\title{
Real-time quantitative PCR for analysis of genetically mixed infections of malaria parasites: technique validation and applications
}

\author{
Sandra J. Cheesman*, Jacobus C. de Roode, Andrew F. Read, Richard Carter \\ Division of Biological Sciences, Institute of Cell, Animal and Population Biology, Ashworth Laboratories, \\ University of Edinburgh, The King's Buildings, West Mains Road, Edinburgh EH9 3JT, UK
}

Received 31 March 2003; received in revised form 25 June 2003; accepted 27 June 2003

\begin{abstract}
A technique that can distinguish and quantify genetically different malaria parasite clones in a mixed infection reliably and with speed and accuracy would be very useful for researchers. Many current methods of genotyping and quantification fall down on a number of aspects relating to their ease of use, sensitivity, cost, reproducibility and, not least, accuracy. Here we report the development and validation of a method that offers several advantages in terms of cost, speed and accuracy over conventional PCR or antibody-based methods. Using real-time quantitative PCR (RTQ-PCR) with allele-specific primers, we have accurately quantified the relative proportions of clones present in laboratory prepared ring-stage mixtures of two genetically distinct clones of the rodent malaria parasite Plasmodium chabaudi chabaudi. Accurate and reproducible measurement of the amount of genomic DNA representing each clone in a mixture was achieved over 100-fold range, corresponding to $0.074 \%$ parasitised erythrocytes at the lower end. To demonstrate the potential utility of this method, we include an example of the type of application it could be used for. In this case, we studied the growth rate dynamics of mixed-clone infections of P. chabaudi using an avirulent/virulent clone combination (AS (PYR) and AJ) or two clones with similar growth rate profiles (AQ and $\mathrm{AJ})$. The modification of the technique described here should enable researchers to quickly extract accurate and reliable data from in-depth studies covering broad areas of interest, such as analyses of clone-specific responses to drugs, vaccines or other selection pressures in malaria or other parasite species that also contain highly polymorphic DNA sequences.
\end{abstract}

(C) 2003 Elsevier B.V. All rights reserved.

Keywords: Plasmodium chabaudi; Real-time quantitative PCR; Parasite clone

\section{Introduction}

Quantitative information on the genetic composition of the parasites present in malaria infections is important in many situations. For example, in the study of mixed infections containing drug-resistant and drug-sensitive parasites, in studies designed to investigate the competitive fitness of avirulent versus virulent genotypes, in vaccine trials, or in studies aimed at investigating 'strain-specific' immune responses to different parasite clones.

To date, progress on fundamental studies of this nature, has been hampered by the lack of a reliable and robust technique that can rapidly distinguish and accurately quantify genetically distinct parasite clones of the same species present in a mixed infection. Existing methods have significant drawbacks: monoclonal antibody-based methods are

\footnotetext{
* Corresponding author. Tel.: +44-131-650-5547.

E-mail address: sandy.cheesman@ed.ac.uk (S.J. Cheesman).
}

able to distinguish different parasites clones by virtue of their variant surface antigens, but, in order to be quantitative, they require labour intensive microscopic analysis using reagents that are often in scarce supply. Standard PCR, although sensitive, cannot reliably quantify mixed genotype infections as it is based on the analysis of end-point amplification products [1].

Real-time quantitative PCR (RTQ-PCR) circumvents the limitations imposed by standard PCR by identifying the position in the PCR reaction where the amount of DNA increases logarithmically from just above background to the plateau phase (called the log-linear phase). Continuous monitoring of PCR samples by fluorescence then allows the small fraction of cycles that fall within the log-linear range to be used to provide reliable information on the initial template concentration.

Many application-specific RTQ-PCR instruments and fluorescence detection systems are now available for research and diagnostics [2]. These packages offer real advantages over standard PCR in terms of their user-friendliness, speed 
of operation and sensitivity of measurement. With the ability to quantify accurately over six to seven orders of magnitude, many systems are now capable of quantifying the amount of targeted DNA sequence to less than 1000 copies. This has made accurate quantification of very low parasite densities [3] as well as gene expression studies possible [4].

We report here, an adaptation to RTQ-PCR that has made it possible to measure accurately the relative proportions of parasites carrying different alleles of the same gene in mixed infections of genetically distinct clones of $P$. chabaudi chabaudi. The experiments described in this paper have two aims: (1) to test the ability of the technique to accurately and reliably quantify the proportions of two genetically distinct clones in a laboratory prepared mixture containing known proportions of each clone, and (2) provide a working example of the type of biological question that could be addressed by this technique. In the latter case, we have analysed the growth rate profiles of a virulent and avirulent clone combination (AJ:AS (PYR)) or a virulent/virulent clone combination (AJ:AQ) in laboratory mice. Although this method was developed for use with the $P$. chabaudi rodent malaria model for the purpose of studying clone-specific immune or drug-responses, the technology should be readily transferable to human malaria parasites or other parasite species whose genetic material contains a high degree of DNA sequence polymorphism.

For researchers interested in using this technique to determine the relative proportions of genetically distinct malaria clones present in clinical samples, however, an important point should be noted; because quantitation depends on use of allele-specific primers as the defining points of allelic variation, prior knowledge of all the clone genotypes present in a sample would be an essential prerequisite to ensure that unknown or novel parasite variants did not escape detection. Use of a primary PCR-based typing technique prior to quantitation may help circumvent such problems $[5,6]$.

\section{Material and methods}

\subsection{Preparation of blood mixtures from individual mice and DNA extraction}

The origins of the cloned lines of $P$. chabaudi chabaudi "isolates" AS and AJ and the pyrimethamine-resistant clone AS (PYR) used in this study are described in $[7,8]$. Blood samples were collected from 5- to 8-week-old inbred female CBA mice infected with AS (PYR) or AJ clones. The parasite clones were harvested at the ring stage of the developmental cycle prior to DNA synthesis [9], to try to ensure that each parasite contained a single haploid genome.

The percentage parasitaemia of each clone was $7.4 \%$ for AS (PYR) and $18.7 \%$ for AJ as determined by microscopic analysis of Giemsa-stained thin blood smears obtained from mouse-tail blood. Red blood cell numbers in each sample were calculated by flow cytometry using a Coulter counter.
Blood samples infected with AS (PYR) or AJ clones were collected by exsanguination of individual mice under general anaesthesia and diluted in citrate saline, $\mathrm{pH}$ 7.2. The blood samples were then adjusted using blood taken from an uninfected mouse so that each sample contained the same number of parasites with the same red blood cell density $\left(7.4 \times 10^{7}\right.$ parasites in $\left.1 \times 10^{9} \mathrm{RBC}\right)$. These two suspensions, containing equal numbers of AS (PYR) or AJ parasite clones were mixed in different proportions in replicate ranging from $0.25 \%$ AS (PYR): $99.75 \%$ AJ through to $99.75 \%$ AS (PYR): $0.25 \%$ AJ. Blood samples were centrifuged briefly to pellet the erythrocytes, and the supernatant removed before the samples were frozen at $-70^{\circ} \mathrm{C}$ prior to DNA extraction. Genomic DNA was extracted using the High Pure PCR Template Preparation Kit (Roche).

\subsection{Preparation of blood mixtures from mouse-tail blood and DNA extraction}

The percentage parasitaemia of each clone was determined by microscopic analysis of Giemsa-stained thin blood smears obtained from mouse-tail blood at $31 \%$ for AS (PYR) and $33.9 \%$ for AJ. Thirty microliters of AS (PYR) and $27 \mu \mathrm{l}$ of AJ-infected mouse-tail blood was diluted to a final volume of $1500 \mu \mathrm{l}$ in citrate saline. Each solution was used to prepare mixtures of AS (PYR) and AJ clones at ratios of 1:99, 10:90, 50:50, 90:10 and 99:1. Ten-fold and 100-fold dilution of these mixtures were prepared using uninfected blood from a control mouse as diluent to represent parasitaemias of 3 and $0.3 \%$. Blood samples were centrifuged briefly to pellet the erythrocytes, and the supernatant removed before the samples were frozen at $-70^{\circ} \mathrm{C}$ prior to DNA extraction using Instagene Matrix (BioRad).

\subsection{Design of allele-specific primers}

The primers used in these experiments were designed to amplify a fragment of the Merozoite Surface Protein 1 (MSP-1) of P. chabaudi chabaudi clones AS ([10]; accession no. L22982) AJ or AQ (Cheesman and Carter, unpublished sequence data). The MSP-1 gene, located on chromosome 8 [11] contains regions of high sequence diversity between clones that facilitate the design of allele-specific primers that can act as clone-specific genetic markers. The primer pairs were designed to yield PCR products of similar sizes for AS (PYR), AJ and AQ alleles. Many of the primers used during the course of this work lacked the high degree of specificity required for RTQ-PCR and were discarded. The following primers produced allele-specific amplicons free of primer-dimer or non-specific product contaminants. AS (PYR) forward and reverse primers sequences: $5^{\prime}$-ACAGTAACACAAGAAGGAAC- $3^{\prime}$ and $5^{\prime}$-GATACTTGTGTTGATGCTGG- $3^{\prime}$. AJ forward and reverse primer sequences: $5^{\prime}$-ACCAGCACAAGAAGCAACAA- $3^{\prime}$ and $5^{\prime}$-TTGCGGGTTCTGTTGAGGCT- $3^{\prime}$. For the AQ and $\mathrm{AJ}$ reactions a different set of $\mathrm{AJ}$ primers were used: 
AJ forward: $5^{\prime}$-ACTGAAGCAACAACACCAGC- $3^{\prime}$ and AJ reverse: 5'-GTTGTTGATGCACTTGCGGGTTC-3'. AQ forward and reverse primer sequences: $5^{\prime}$-TTACACCCCAAACCGCTAC-3' and 5'-GTAGTTGTTCCTGTGGCAG-3'. Primers were made by Oswell Research Products Ltd. and were HPLC purified. Primer pairs were designed with the aid of Primerfinder Software (http://eatworms.swmed.edu/ tim/ primerfinder/) to minimise the possibility of self-dimerisation or heterodimerisation that could lead to non-specific PCR amplicons.

\subsection{Preparation of genomic DNA standards for $R T Q-P C R$}

DNA standards were prepared by the following method to increase the quantity of genomic DNA recovered so that highly concentrated stocks of standard were available for long-term use.

Whole blood samples infected with AS (PYR) or AJ parasite clones were collected from five mice and used to prepare genomic DNA [7]. Briefly, mouse white blood cells were removed by column purification using CF11 (Sigma) and Plasmodipur $^{\mathrm{TM}}$ filters (Euro-Diagnostica). Parasites were isolated from host erythrocytes by saponin lysis and genomic DNA prepared by phenol-chloroform extraction. DNA standards were treated with RNAse before re-extracting with phenol-chloroform. The purity and quantity of the DNA was determined by fluorimetry, UV spectrophotometry, and electrophoresis of serially diluted ethidium-bromide stained samples on agarose gels. Standards consisting of 10-fold serial dilutions of genomic DNA were freshly prepared from concentrated stocks for each RTQ-PCR experiment.

Accurate quantification of the clones present in the mixed infection experiments described here necessitated very careful measurement and dilution of each standard (AS (PYR), $\mathrm{AJ}$ and $\mathrm{AQ}$ ) to ensure they were close to equal in their DNA concentration. We found that fluorometric analysis was the most reliable determinant of DNA concentration and UV spectrophotometry the best determinant of DNA quality.

\section{5. $P C R$}

PCR was performed using a Roche LightCycler [12]. Ten microliter reactions were set up using FastStart DNA Master SYBR Green I kit reagents according to the manufacturers instructions in LightCycler capillaries (Roche).

Prior to the work described here, reaction components and cycling conditions were optimised to give reliable and reproducible results. Magnesium chloride and primer concentration, denaturation, annealing and elongation rates and times were varied to determine the conditions under which only the specific amplicon was produced. The average amplification efficiencies of the reactions were closely matched for each set of standards because small differences can result in large errors in quantification between samples. The average amplification efficiency, $E$, was determined from the slope of the standard curves produced in each experiment using the equation $E=10^{-1 / \text { slope }}$. PCR reactions where the amplicon doubles at every cycle have an optimal efficiency of 2.0 compared to reactions where no amplification occurs and the efficiency is 1.0. Factors that may influence the amplification efficiency include, for example, insufficient optimisation of reaction conditions, poor template quality, poor primer design, or the presence of PCR inhibitors in the reaction.

Reactions were set up with AS- or AJ-specific primer sets containing $0.4 \mu \mathrm{M}$ forward and reverse primers and 4.5 and $3.5 \mathrm{mM} \mathrm{MgCl}_{2}$, respectively. All samples were analysed in replicate within each LightCycler run. The amplification program comprised 40 cycles with the following steps: an initial "Hot Start" at $95^{\circ} \mathrm{C}$ for $600 \mathrm{~s}$ followed by $95^{\circ} \mathrm{C}$ with a $0 \mathrm{~s}$ hold, cooling at $20^{\circ} \mathrm{C} / \mathrm{s}$ to $59^{\circ} \mathrm{C}$ with a $6 \mathrm{~s}$ hold for AS (PYR) or cooling at $20^{\circ} \mathrm{C} / \mathrm{s}$ to $61^{\circ} \mathrm{C}$ with a $6 \mathrm{~s}$ hold for AJ, heating at $20^{\circ} \mathrm{C} / \mathrm{s}$ to $72{ }^{\circ} \mathrm{C}$ with a $7 \mathrm{~s}$ hold. The fluorescent signal produced from the amplicon was acquired at the end of the polymerisation step at $72{ }^{\circ} \mathrm{C}$. Heating at $20^{\circ} \mathrm{C} / \mathrm{s}$ to $95^{\circ} \mathrm{C}$ with $0 \mathrm{~s}$ hold, cooling at $20^{\circ} \mathrm{C} / \mathrm{s}$ to $65^{\circ} \mathrm{C}$ and heating at $0.2^{\circ} \mathrm{C} / \mathrm{s}$ to $95^{\circ} \mathrm{C}$ in a continuous acquisition mode produced the melting curve data.

Samples of a 10-fold serial dilution of AS (PYR) or AJ gDNA in the range $60-0.006 \mathrm{ng}$ were used as quantification standards for the LightCycler calibration curve. Samples prepared from artificial mixtures of parasite clones were analysed in replicate within the same run. Maximum recovery filter tips (AXYGEN ${ }^{\circledR}$ Scientific) and "No Stick" microtubes (Alpha Laboratories) were used in these experiments. LightCycler data were analysed by the Fit Points method.

Reactions that were set up to measure the proportion of $\mathrm{AJ}$ and $\mathrm{AQ}$ clones present in a sample used the same reaction conditions as described for the AS (PYR) and AJ clones except $4 \mathrm{mM} \mathrm{MgCl} 2$ was used for $\mathrm{AQ}$ and $3.5 \mathrm{mM} \mathrm{MgCl} 2$ for $\mathrm{AJ}$ reactions. The annealing temperature used for $\mathrm{AJ}$ and AQ reactions was $63{ }^{\circ} \mathrm{C}$ with a $6 \mathrm{~s}$ hold.

\subsection{P. chabaudi mixed-clone infection experiments}

Two sets of three CBA female mice were infected with a mixture consisting of $1 \times 10^{6}$ parasites of clones of AS (PYR) and AJ (Group 1) or $2.5 \times 10^{6}$ parasites of clones AQ and AJ (Group 2). Each Group 1 mouse received a total of $2 \times 10^{6}$ parasites and each Group 2 mouse $5 \times$ $10^{6}$ parasites. Parasitaemias were monitored and recorded over the course of the infection by microscopic analysis of Giemsa-stained thin blood smears. Five microliters of tail blood was collected from Group 1 mice and $10 \mu$ l of tail blood from Group 2 mice into citrate saline on days 3-8 post-infection. The samples were centrifuged briefly and the RBC pellets stored at $-70^{\circ} \mathrm{C}$ prior to DNA extraction using the Instagene Matrix (BioRad) kit for AS (PYR) and AJ samples and the High Pure PCR Template Preparation Kit (Roche) for AQ and AJ samples. 


\section{Results}

\subsection{Allele-specific melting peaks and cross-reaction tests}

A critical assumption underlying these experiments is that the primers used to detect AS (PYR) and AJ MSP-1 alleles are specific for each clone. To test this we conducted a cross-reaction test where AS (PYR) genomic DNA was amplified with AS- and AJ-specific primers, and AJ genomic DNA was amplified with AJ- and AS-specific primers
(Fig. 1a and b). These results show the allele-specific melting peak produced by each clone. Only the clone-specific allele was amplified in each case. Use of the 'wrong' primer set produced either no signal or melting peaks characteristic of primer-dimer formation. No non-specific PCR products were produced when the correct clone-specific primers were used, as judged by analysis of the melting peaks produced by the LightCycler software. Cross-reaction tests were also conducted on AQ and AJ DNA; in each case only the correct allele-specific melting peak was detected (data not shown).
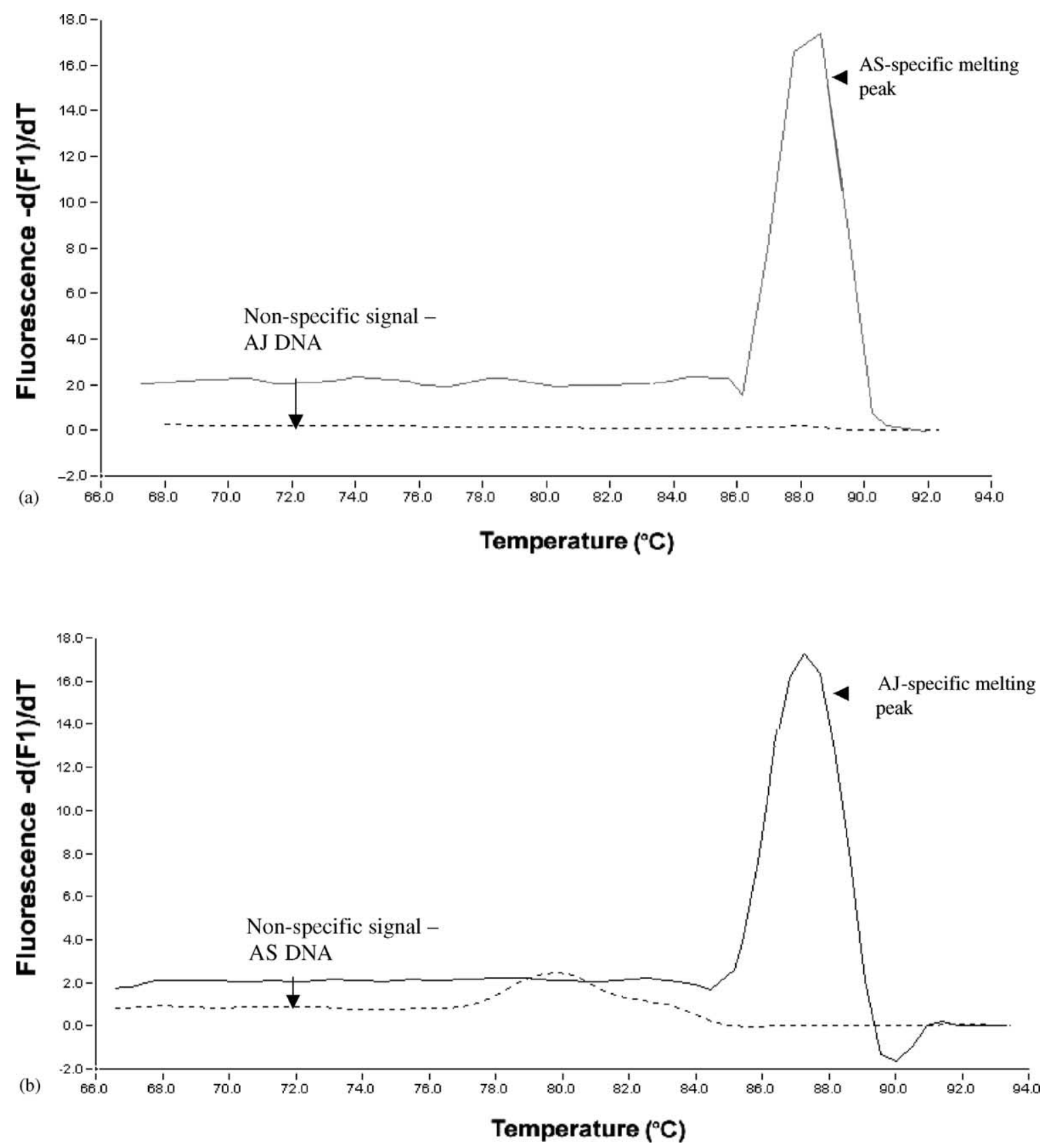

Fig. 1. Fluorescence melting curve data derived from cross-reaction tests performed to confirm the specificity of AS (PYR) and AJ primers for their target sequence. (a) The AS-specific melting peak produced by the pure AS (PYR) clone using AS-specific primers and the signal produced using pure AJ genomic DNA. (b) The AJ-specific melting peak produced by the pure AJ clone using AJ-specific primers and the non-specific signal produced using AJ-specific primers with pure AS (PYR) genomic DNA. 
Table 1

The proportions of AS (PYR) and AJ clones quantified by RTQ-PCR from duplicate mixtures (Experiments 1 and 2) containing different proportions of AS (PYR) and AJ blood-stage parasite clones

\begin{tabular}{|c|c|c|c|c|c|c|c|}
\hline \multicolumn{2}{|l|}{ Expected } & \multicolumn{2}{|c|}{ Measured in Experiment 1} & \multicolumn{2}{|c|}{ Measured in Experiment 2} & \multicolumn{2}{|c|}{ Mean of Experiments 1 and 2} \\
\hline$\%$ AS clone & $\% \mathrm{AJ}$ clone & $\%$ AS clone & $\% \mathrm{AJ}$ clone & $\%$ AS clone & $\%$ AJ clone & $\%$ AS clone & $\% \mathrm{AJ}$ clone \\
\hline 0 & 100 & 0.00 & 100.00 & 0.00 & 100.00 & 0.00 & 100.00 \\
\hline 0.25 & 99.75 & 0.00 & 100.00 & 0.69 & 99.31 & 0.35 & 99.65 \\
\hline 0.50 & 99.5 & 0.38 & 99.62 & 0.78 & 99.22 & 0.58 & 99.42 \\
\hline 1 & 99 & 0.00 & 100.00 & 0.00 & 100.00 & 0.00 & 100.00 \\
\hline 2 & 98 & 2.03 & 97.97 & 1.97 & 98.03 & 2.00 & 98.00 \\
\hline 6 & 94 & 0.00 & 100.00 & 5.81 & 94.19 & 2.90 & 97.10 \\
\hline 20 & 80 & 18.31 & 81.69 & 19.07 & 80.93 & 18.69 & 81.31 \\
\hline 32.5 & 67.5 & 32.68 & 67.32 & 33.84 & 66.16 & 33.26 & 66.74 \\
\hline 50 & 50 & 46.16 & 53.84 & 46.23 & 53.77 & 46.20 & 53.80 \\
\hline 67.5 & 32.5 & 66.33 & 33.67 & 68.97 & 31.03 & 67.65 & 32.35 \\
\hline 80 & 20 & 81.38 & 18.62 & 74.07 & 25.93 & 77.72 & 22.28 \\
\hline 94 & 6 & 93.86 & 6.14 & 92.65 & 7.35 & 93.26 & 6.74 \\
\hline 98 & 2 & 97.75 & 2.25 & 98.39 & 1.61 & 98.07 & 1.93 \\
\hline 99 & 1 & 98.96 & 1.04 & 98.70 & 1.30 & 98.83 & 1.17 \\
\hline 99.5 & 0.5 & 100.00 & 0.00 & 99.34 & 0.66 & 99.67 & 0.33 \\
\hline 99.75 & 0.25 & 99.62 & 0.38 & 99.50 & 0.50 & 99.56 & 0.44 \\
\hline 100 & 0 & 100.00 & 0.00 & 100.00 & 0.00 & 100.00 & 0.00 \\
\hline 0 & 0 & 0.00 & 0.00 & 0.00 & 0.00 & 0.00 & 0.00 \\
\hline
\end{tabular}

\subsection{Measurements of MSP-1 AS and AJ alleles in laboratory prepared blood mixtures containing known proportions of each clone}

Mouse blood infected with $7.4 \times 10^{7} \mathrm{ml}^{-1}$ of parasites representing clones of $P$. chabaudi chabaudi AS (PYR) or AJ both at an RBC density of $1 \times 10^{9} \mathrm{RBC} / \mathrm{ml}$ (i.e. $7.4 \%$ parasitaemia) was used to make duplicate artificial mixtures containing different proportions of each clone. The proportions of the mixtures tested ranged from 0.25 to $100 \%$ AS (PYR) or AJ. In the lower range, for example, mixtures comprised $0.25 \%$ AS (PYR) and $99.75 \%$ AJ or the reverse, $0.25 \%$ AJ and $99.75 \%$ AS (PYR).

Table 1 shows the actual proportions obtained by RTQ-PCR for duplicate Experiments 1 and 2, together with the mean value obtained for both experiments. The results show that the mean proportions of AS (PYR) and AJ clones quantified in both experiments correlates well with the proportions expected from the mixtures. Data from the individual experiments show, however, that some of the reactions measuring in the lower ranges occasionally produced no specific PCR product (e.g. in Experiment 1 the value expected for AS (PYR) of $0.25 \%$ was quantified at $0 \%)$. Given that the accuracy of measurement in the higher ranges (above $2 \%$ ) is very good, we attribute such discrepancies to the errors involved in measuring and dispensing very small volumes accurately during the actual preparation of the blood mixtures themselves.

All samples analysed in this experiment, with the exception of the standards, contain mouse host nucleated cell DNA. To ensure that both primer sets did not cross-react with the mouse DNA, we also analysed samples containing no parasites. No amplicon was produced by either set of AS (PYR) or AJ primers from such material (Table 1). The standard curves produced for AS (PYR) and AJ DNA in these experiments were linear over the four-log range of DNA concentration from $6 \mathrm{pg}$ to $60 \mathrm{ng}$ that was used (data not shown). The average amplification efficiencies $(E)$ for both sets of standards were checked to ensure that they were comparable in order to reduce the likelihood of quantification bias in the parasite mixtures analysed here. The average amplification efficiencies were calculated at 1.978 for AS (PYR) and 1.980 for AJ.

\subsection{Sensitivity of measurement}

We investigated whether the technique was sensitive enough to accurately quantify material containing a 50:50 mixture of AS (PYR) and AJ clones over two orders of magnitude below those used in the initial experiments (i.e. corresponding to $7.4,0.74$ and $0.074 \%$ parasitaemia or $74,000,7400$ and 740 parasites in the fraction of the sample analysed). The results, shown in Table 2, demonstrate that even at the lowest level tested (740 parasites), the deviation from the expected value was less than $11 \%$. We also tested whether using DNA samples prepared by a less expensive, quicker method (Instagene Matrix (BioRad)) using small quantities of mouse-tail blood would also produce accurate results on the LightCycler (Roche) system. For this experiment, artificial blood mixtures were prepared from mouse-tail blood in the proportions AS:AJ; 1:99, 10:90, 90:10, 99:1 and 50:50. These suspensions were used to provide a 10- and 100-fold dilution series to represent parasitaemias of 30,3 and $0.3 \%$, respectively. DNA was extracted from these samples using Instagene Matrix (BioRad). Each sample was quantified twice and the proportion 
Table 2

RTQ-PCR values obtained from genomic DNA samples extracted from blood mixtures derived from individual mice containing equal numbers of parasite clones AS (PYR) and AJ over 100-fold range

\begin{tabular}{|c|c|c|c|c|c|}
\hline $\begin{array}{l}\text { Calculated total number of AS } \\
\text { or AJ parasites in fraction of } \\
\text { sample analysed }\end{array}$ & $\begin{array}{l}\text { Mean concentration of } \\
\text { AS clone detected }(\mathrm{ng} / \mu \mathrm{l})\end{array}$ & $\begin{array}{l}\text { Mean concentration of } \\
\text { AJ clone detected }(n g / \mu l)\end{array}$ & $\begin{array}{l}\text { Expected } \% \\
\text { AS or AJ }\end{array}$ & $\begin{array}{l}\text { Mean \% AS } \\
\text { detected }\end{array}$ & $\begin{array}{l}\text { Mean \% AJ } \\
\text { detected }\end{array}$ \\
\hline 74000 & 0.5135 & 0.598 & 50 & 46.2 & 53.8 \\
\hline 7400 & 0.032 & 0.03165 & 50 & 50.3 & 49.7 \\
\hline 740 & 0.007315 & 0.00594 & 50 & 55.2 & 44.8 \\
\hline
\end{tabular}

of AS (PYR) measured was based on the mean of these experiments. The observed proportion of AS (PYR) was then plotted against the expected proportion of AS (PYR), as shown in Fig. 2. The results show that DNA extracted from mouse-tail blood using Instagene Matrix gives accurate estimates of the proportions of AS (PYR) and AJ clones present in the artificial mixtures, over a range of 30, 3 and $0.3 \%$ parasitaemia. All data points fit almost on a straight line of expected values.

\subsection{Analysis of between run variation}

In the experiments described so far we have shown that RTQ-PCR using allele-specific primers is both accurate and sensitive. We also investigated how robust the method was in terms of how well random errors within the system appear to be controlled between runs. Ten-fold serial dilutions (10- to 100,000-fold) of $P$. chabaudi AJ and AQ DNA were made on five independent occasions. Each dilution series was amplified with AQ- or AJ-specific MSP-1 primers on the LightCycler (Roche) in five independent runs. The Crossing Points, defined as the position in the reaction where the fluorescence of the PCR products become greater than the background signal from the SYBR Green 1 fluorescent dye, for each series were calculated using the LightCycler Soft-

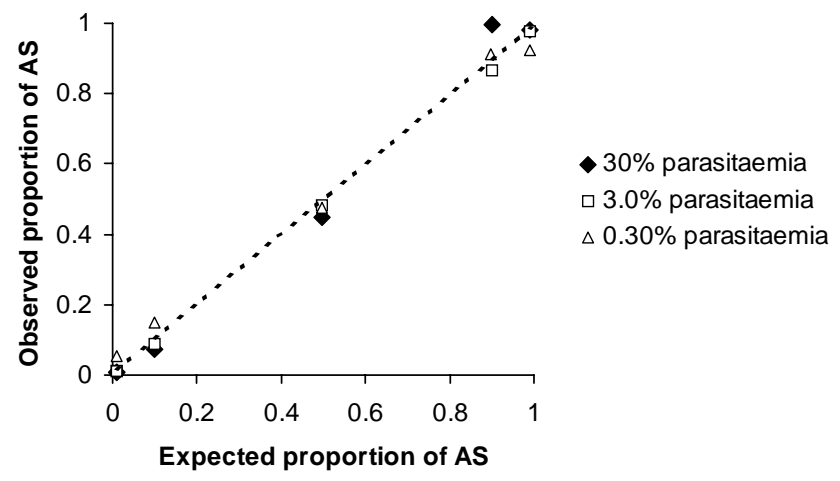

Fig. 2. Analysis of the sensitivity of the RTQ-PCR technique using artificial mixtures containing known proportions of AS (PYR) and AJ clones obtained from mouse-tail blood. Artificial mixtures containing AS (PYR) and AJ parasite clones were prepared in these proportions: AS:AJ; 50:50, 10:90, 1:99, 99:1 and 90:10. Each mixture was further diluted to make artificial mixtures representing 30,3 and $0.3 \%$ parasitaemia in the same proportions. The observed proportion of AS (PYR) detected is plotted against the expected proportion of AS (PYR). ware. Fig. $3 a$ and $b$ shows the mean values and standard deviation of each dilution series of AQ and AJ DNA. Both curves are linear over a five-log range. The percentage relative standard deviation $(\%$ rsd $=($ standard deviation $/$ mean $)$ $\times 100$ ), a measure of the overall precision of the method was calculated at $<3.6 \%$ for AQ and $<2.3 \%$ for AJ DNA across the sample dilution range, indicating that the method is extremely robust across a wide range of dilutions for both clones.

\subsection{Real-time quantitative PCR analysis of mixed-clone infections of $A S$ and $A J$ or $A Q$ and $A J$ in laboratory mice}

Mixed infections of AS (PYR) and AJ (Fig. 4a) and AQ and AJ clones (Fig. 4b) were analysed using RTQ-PCR in three individual mice per group. The initial inoculum contained a 50:50 mixture of each clone, i.e. AS and AJ (Fig. 4a) or AQ and AJ (Fig. 4b). For the Group 1 mice, AJ rapidly dominated the infection over AS (PYR), but the proportion remained stable between the 3rd and 8th day of infection in all three mice. In the AJ $+\mathrm{AQ}$ mixture, $\mathrm{AJ}$ also dominated the infection, but less than in the AS + AJ mixture, and the proportions also remained stable between days 3 and 8 post-infection.

In both examples of mixed-clone infections, viz. $\mathrm{AS}+\mathrm{AJ}$ and $\mathrm{AJ}+\mathrm{AQ}$, the proportions of each clone remained remarkably constant during the course of infection and between replicate infections in different mice. This is particularly striking, given the variation observed in terms of the day and peak level of parasitaemia, as was especially noticeable for the AJ + AQ combination (Fig. 4b). The observation suggests a rather tight biological and genetic control over the dynamic behaviour of the parasite clones in a malaria infection. Use of isogenic lines of laboratory mouse with cloned 'strains' of rodent malaria parasite has revealed biological phenomena that could not have been detected in natural human malaria infections.

\section{Discussion}

The aim of these experiments was to establish if we could adapt RTQ-PCR to accurately identify and measure the proportions of two genetically distinct clones of the rodent malaria parasite $P$. chabaudi chabaudi in a laboratory prepared blood-stage infection. This approach has not, to our 

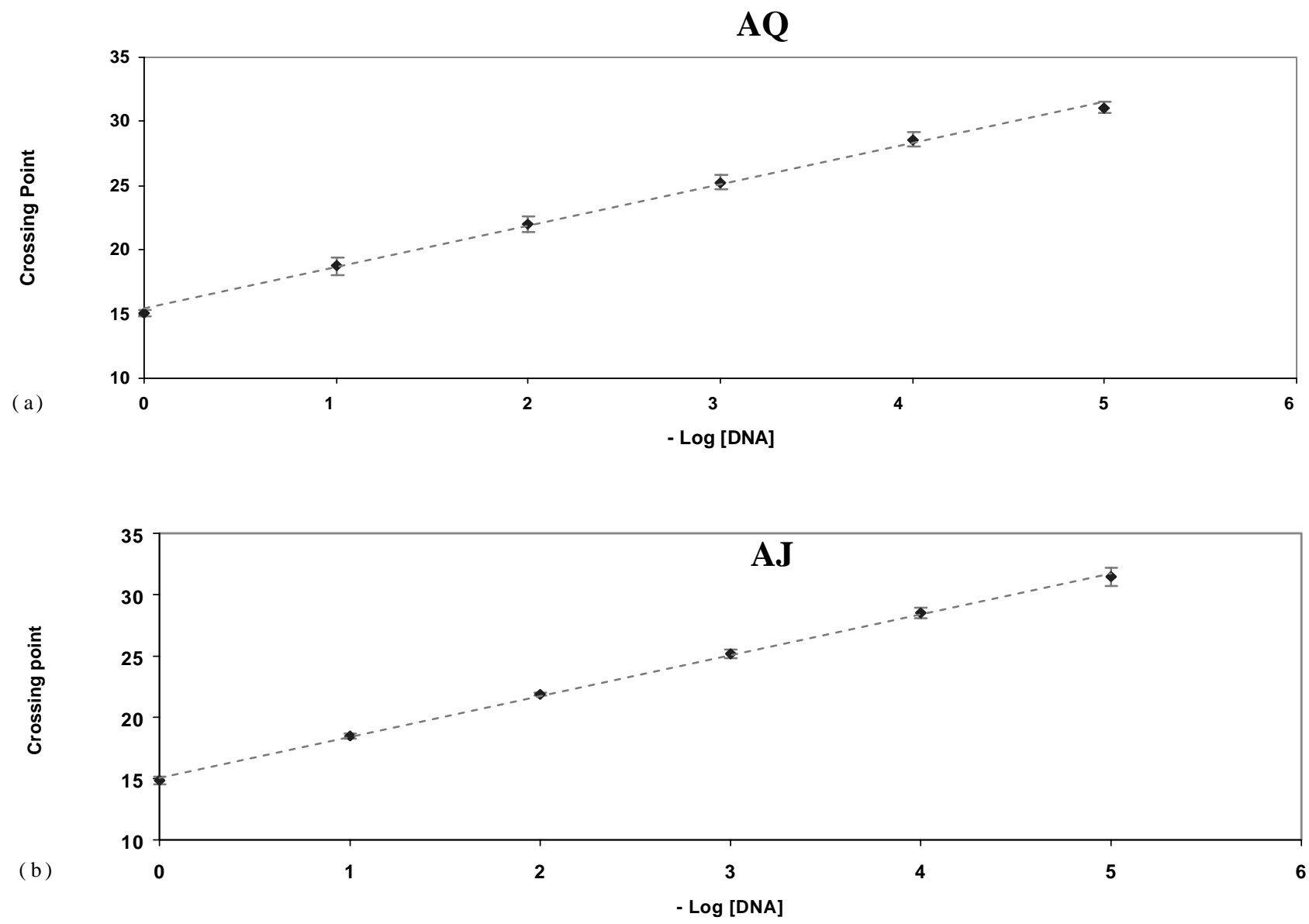

Fig. 3. Reproducibility of the RTQ-PCR using MSP-1 AJ or AQ allele-specific primers. Five 10-fold serial dilutions of AJ or AQ DNA templates were prepared and amplified on five independent occasions. The means of the five Crossing Points are plotted for each concentration of DNA used. Error bars represent the standard deviation of each dilution within the series. The precision of the measurements, expressed as the percentage relative standard deviation (\% rsd) was calculated at (AQ): undiluted DNA sample, 1.46\%; 1/10 dilution, 3.62\%; 1/100 dilution, 2.65\%; 1/1000 dilution, 2.06\%; 1/10,000 dilution, $1.82 \%$; 1/100,000 dilution, $1.43 \%$. (AJ): Undiluted DNA sample, $2.10 \%$; $1 / 10$ dilution, $1.13 \%$; $1 / 100$ dilution, $0.54 \%$; $1 / 1000$ dilution, $1.39 \%$; $1 / 10,000$ dilution, $1.50 \%$; and 1/100,000 dilution, $2.35 \%$.

knowledge, been attempted before in this context, and was made possible by the use of primers designed against MSP-1 gene sequences containing large regions of variability between the clones used in this study, AS (PYR), AJ and AQ.

Artificial blood mixtures containing a calculated number of parasite clones demonstrated that the technique is accurate and sensitive enough to measure over a wide range of different proportions ranging from $0.25 \%$ AS (PYR) and $99.75 \% \mathrm{AJ}$ or the reverse, $0.25 \% \mathrm{AJ}$ and $99.75 \%$ AS (PYR).

We used two different methods to prepare DNA for these experiments to determine whether the real-time method required highly purified template DNA such as was made using the High Pure PCR Template Preparation Kit (Roche) for accuracy or would give comparable results with templates prepared by less rigorous methods using smaller blood volumes (Instagene Matrix (BioRad)).

LightCycler data derived from DNA samples prepared by both methods gave results that were accurate over two orders of magnitude below the starting concentration.
The precision of the technique was tested over a five orders of magnitude dilution series with $\mathrm{AQ}$ and $\mathrm{AJ}$ genomic DNA diluted and amplified on five separate occasions. The percent relative standard deviation, even at the greatest dilution $(1 / 100,000)$ was only $1.43 \%$ for AQ and $2.35 \%$ for AJ, indicating that the technique is repeatable over a wide range of DNA concentration. The precision across the whole dilution series was calculated to be less than $4 \%$ rsd for AQ primers and less than $2.5 \%$ rsd for $\mathrm{AJ}$ primers.

To demonstrate an application of the technique, we investigated the dynamics of mixed infections of $P$. chabaudi clones $\mathrm{AJ}$ with $\mathrm{AQ}$ or $\mathrm{AJ}$ with $\mathrm{AS}$ (PYR) in inbred CBA female mice. The starting inocula consisted of a 50:50 mixture of the parasites of each clone. In the AJ and AQ mixture, there was a moderate predominance of $\mathrm{AJ}$ over $\mathrm{AQ}$, leading to about $70 \% \mathrm{AJ}$ by day 8 of infection. In the $\mathrm{AJ}$ and AS (PYR) mixture, the predominance of AJ over AS (PYR) was much stronger leading to $90-95 \%$ AJ by day 8 of the infection. In each combination, there was remarkable consistency in the behaviour of the mixed clones between 

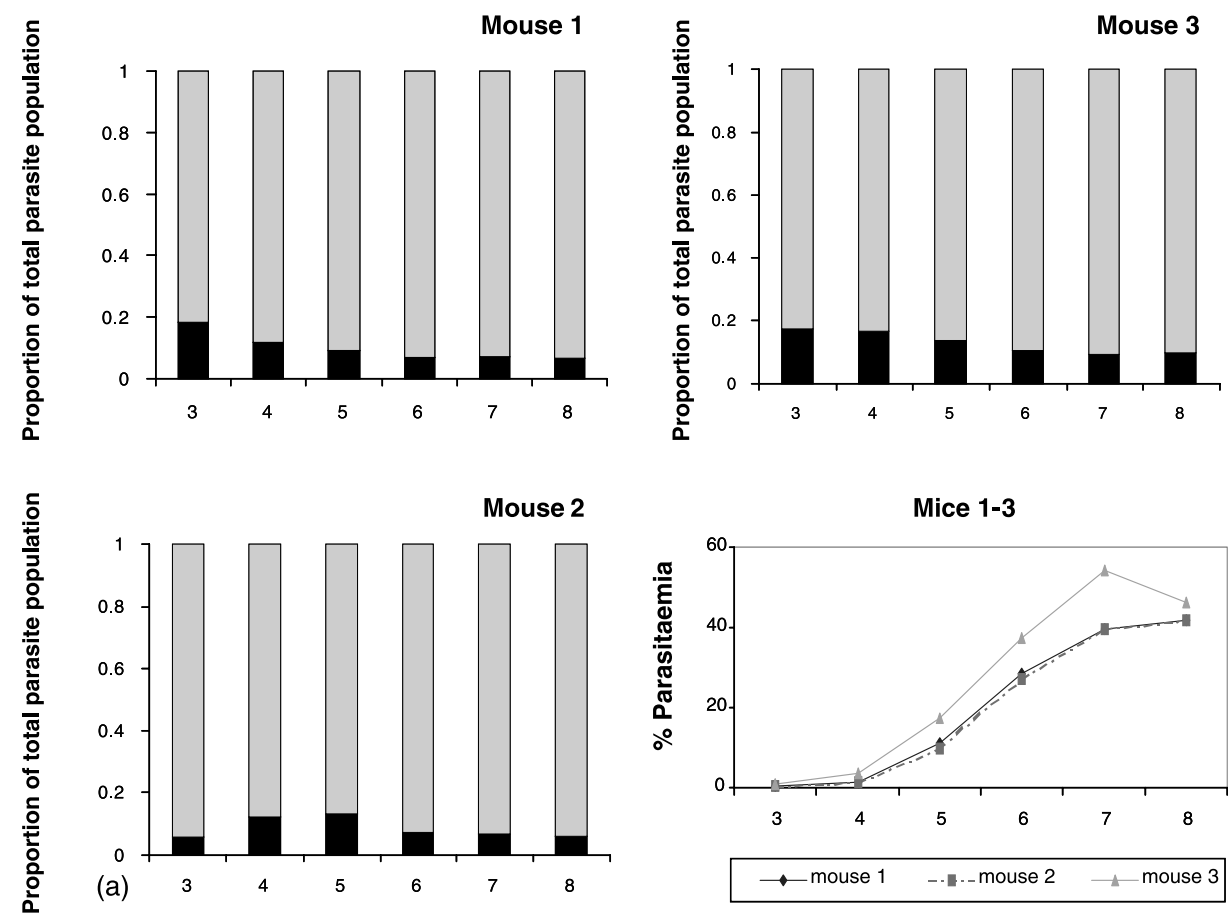

Day post-infection

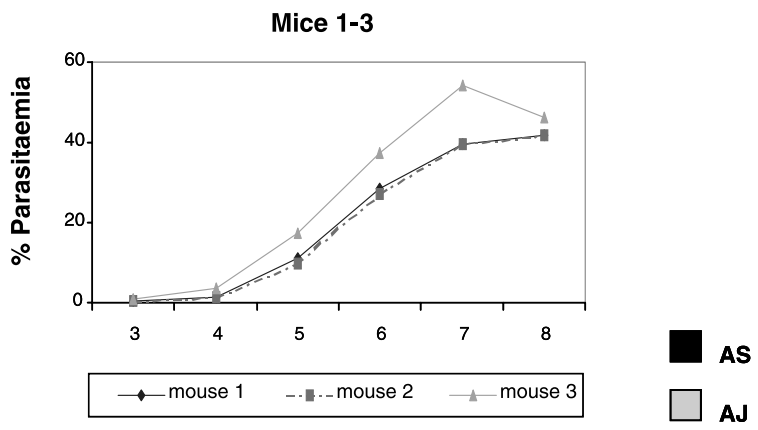

Day post-infection
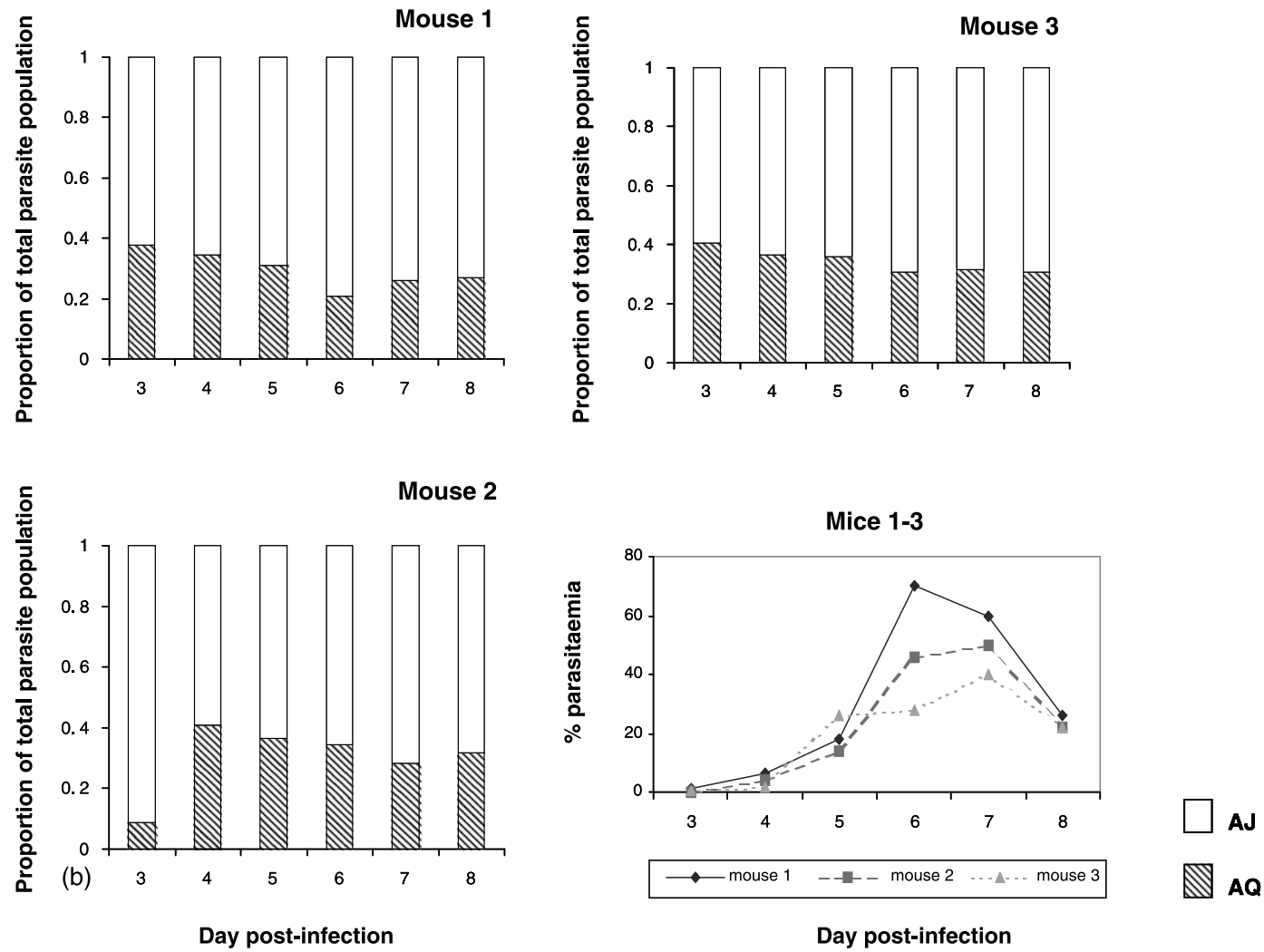

Fig. 4. The mixed infection dynamics of $P$. chabaudi clones AS (PYR) and AJ ((a); Group 1 mice) and AQ and AJ ((b); Group 2 mice) measured using RTQ-PCR. Individual mice were inoculated with a mixture of $1 \times 10^{6}$ parasites of clones AS (PYR) and AJ (Group 1$)$ or $2.5 \times 10^{6}$ parasites of clones of AQ and AJ (Group 2) representing a total of $2 \times 10^{6}$ or $5 \times 10^{6}$ clones, respectively. (a) shows the proportion of parasite clones quantified for AS (PYR) and AJ, and (b) AQ and AJ clones at days 3-8 post-infection together with the corresponding parasitaemia for the course of each infection. 
individual infections. The relationships between the growth of AS (PYR) and AJ in the mixtures reflects the difference in growth rate we have observed between these two clones when examined in single-clone infection situations, with AJ consistently outgrowing AS (data not shown). Our data shows that AS (PYR) grows even more slowly than expected when in competition with AJ. The method is thus shown to be able to explore behaviour of the parasites not previously available to observation.

Having verified in these pilot experiments, the utility of the RTQ-PCR adaptation using AS (PYR), AJ and AQ clones of $P$. chabaudi, we have also confirmed the general applicability of the technique to other clone combinations (e.g. AS (PYR) and $\mathrm{CB}$ and $\mathrm{AJ}$ and $\mathrm{CB}$ clones of $P$. chabaudi chabaudi, (results not shown). It is now our intention to use this technique to genotype and quantify the relative proportions of parasites present in mixed-clone infections of $P$. chabaudi within the context of 'strain-specific' immunity and drug-resistance. Our adaptation of the standard RTQ-PCR technique using allele-specific primers, should also provide scope for use with other malaria parasite species in more diverse situations.

\section{Acknowledgements}

We would like to thank Les Steven and Richard Culleton for their assistance in working with $P$. chabaudi infections in mice, Judi Allen and colleagues for use of the LightCycler instrument, Lisa Ranford-Cartwright and Andy Bell for their invaluable help and advise on LightCycler issues and Richard Culleton for critical reading of the manuscript. This work was supported by grants from the Wellcome Trust and BBSRC. J.C. de Roode was supported by the Darwin Trust of Edinburgh.

\section{References}

[1] Makrigiogos GM, Chakrabarti S, Zhang Y, Kaur M, Price BD. A PCR-based amplification method retaining the quantitative difference between two complex genomes. Nat Biotechnol 2002;20: 936-9.

[2] Bell AS, Ranford-Cartwright L. Real-time quantitative PCR in parasitology. Trends Parasitol 2002;18:337-42.

[3] Polanco J, Rodriguez JA, Corredor V, Patarroyo MA. Plasmodium vivax: parasitemia determination by real-time quantitative PCR in Aotus monkeys. Exp Parasitol 2002;100:131-4.

[4] Blair PL, Witney A, Haynes JD, Moch JK, Carucci DJ, Adams JH. Transcripts of developmentally regulated Plasmodium falciparum genes quantified by real-time RT-PCR. Nucleic Acids Res 2002;30:2224-31.

[5] Contamin H, Fandeur T, Bonnefoy S, Skouri F, Ntoumi F, Mercereau-Puijalon O. PCR typing of field isolates of Plasmodium falciparum. J Clin Microbiol 1995;33:944-51.

[6] Anderson TJC, Su X, Bockarie M, Lagog M, Day KP. Twelve microsatellite markers for characterization of Plasmodium falciparum from finger-prick blood samples. Parasitology 1999;119: $113-25$.

[7] Grech K, Martinelli A, Pathirana S, Walliker D, Hunt P, Carter R. Numerous, robust genetic markers for Plasmodium chabaudi by the method of amplified fragment length polymorphism. Mol Biochem Parasitol 2002;123(2):95.

[8] Hayton K, Ranford-Cartwright L, Walliker D. Sulfadoxine-pyrimethamine resistance in the rodent malaria parasites Plasmodium chabaudi. Antimicrob Agents Chemother 2002;46:2482-9.

[9] Newbold CI, Boyle DB, Smith CC, Brown KN. Stage specific protein and nucleic acid synthesis during the asexual cycle of the rodent malaria Plasmodium chabaudi. Mol Biochem Parasitol 1982;5:3344.

[10] McKean PG, O’Dea K, Brown KN. Nucleotide sequence analysis and epitope mapping of the merozoite surface protein 1 from Plasmodium chabaudi chabaudi AS. Mol Biol Parasitol 1993;62:199-210.

[11] Carlton JMR, Vinkenoog R, Waters AP, Walliker D. Gene synteny in species of Plasmodium. Mol Biol Parasitol 1998;93:285-94.

[12] Wittwer CT, Ririe KM, Andrew RV, David DA, Gundry RA, Balis UJ. The LightCycler: a microvolume multi-sample fluorimeter with rapid temperature control. Biotechniques 1997;22:130. 